\title{
We Still Have to Eat: Communication Infrastructure and Local Food Organizing as Public Health Responses to COVID-19 in Greensboro, North Carolina
}

\author{
Marianne LeGreco *, Jasmine Palmer and Marianna Levithan \\ University of North Carolina at Greensboro, Greensboro, NC, United States
}

Food insecurity remains a pervasive and persistent social justice concern, both locally and globally-a concern that was heightened during the COVID-19 pandemic. This essay focuses on three short case studies around local food organizing, communication, and community in Greensboro, NC. Partners across three separate but related interventions leveraged their community and communication resources through listening sessions, surveys, and stories

OPEN ACCESS

Edited by:

Constance Gordon,

San Francisco State University, United States

Reviewed by:

James Olumide Olufowote, University of Oklahoma, United States Benjamin $R$ Bates,

Ohio University, United States

${ }^{*}$ Correspondence: Marianne LeGreco melegrec@uncg.edu

Specialty section: This article was submitted to Health Communication, a section of the journal Frontiers in Communication

Received: 09 May 2021 Accepted: 23 August 2021 Published: 06 September 2021

Citation:

LeGreco M, Palmer J and Levithan M (2021) We Still Have to Eat:

Communication Infrastructure and Local Food Organizing as Public Health

Responses to COVID-19 in

Greensboro, North Carolina.

Front. Commun. 6:707144.

do: $10.3389 /$ fcomm.2021.707144 to ensure that individuals and families could continue to access food during the uncertainty of the COVID-19 pandemic. By offering these case studies as an example of organizing (and reorganizing) during COVID-19, the analysis also opens up a conversation about power, resistance, and change at the intersections of poverty and access. Scholarly discussions of food insecurity continue to reinforce the need to address both food access and poverty in attempts to build resilient food systems. We take a community-engaged approach that emphasizes the importance of communication infrastructure to illustrate both the simple and mundane resources as well as the creative and innovative interventions that communities and their partners implemented during the initial onset of COVID-19 in the United States.

Keywords: food security, community engagement, communication infrastructure, local food organizing, food access, resilience

\section{INTRODUCTION}

In March 2020, when stay-at-home orders and social distancing practices related to COVID-19 began emerging in the U.S., most public health messages emphasized behaviors and practices related primarily to the spread of the disease between individuals and across groups. Wash your hands. Stay six feet apart. Wear a mask in public. Alongside these messages, a similarly-important set of conversations developed around local food systems and the need for community-based and culture-centered health messaging. Health, organizational, and environmental communication scholars were quick to highlight the importance of communication infrastructure in reorganizing health practices (e.g., Dutta et al., 2020), particularly as it related to local food systems and practices (Schraedley et al., 2020).

The need for secure food systems that are accessible and affordable comes into sharp-and sometimes stark-relief during a global pandemic. Grocery stores and their employees were labeled essential. Restaurants closed or converted to curbside and delivery systems. Farmers markets and food pantries scrambled to create contactless and socially-distanced distribution models. School districts enacted networks to get food to students who were now learning at home (Hodgin, 2020). 
From the consumer-side of food system, people hoarded food and resources purchased from grocery stores-particularly meat, non-perishable food items, and we are certain few will ever forget, toilet paper (Kunkle and Ruane, 2020). At the same time, individuals and families sometimes struggled to find food-especially when someone in their household either lost a job or could not work because of how city, county, and/or statelevel policies impacted their place of work (Sy, 2020).

The ways in which local stakeholders adjusted, reorganized, and in some cases created new relationships across food systems served as a reminder that even during a pandemic we still have to eat. As we consider how communication is tied intricately to food systems and practices, particularly during periods of crisis, we find it prudent to document how communities responded to growing concerns around food security at the initial onset of COVID-19.

This essay features three, short case examples that illustrate public health and community-based responses to reorganizing food in Greensboro, NC during COVID-19 stay-at-home orders and initial social-distancing phases. We emphasize the importance of communication infrastructure in a community's capacity to quickly develop public health and food systems interventions during a period of extreme uncertainty and reorganizing, like a global pandemic. After drawing theoretical connections between communication infrastructure, public health interventions, and local food organizing, we focus on how school meal programs, farmers markets, and community food networks relied on existing communication infrastructure to help individuals and families secure food in the midst of a pandemic.

\section{LOCAL FOOD ORGANIZING AND HEALTH COMMUNICATION INFRASTRUCTURE}

To illustrate how public health and food systems interventions apply not only to individual health behaviors related to COVID19 , but also how communities reorganized local food systems and practices amidst the spread of the virus, we ground our work in the importance of communication infrastructure as it relates to health communication messaging and interventions (Kim and Ball-Rokeach, 2006; Dutta and Thaker, 2019). In particular, we emphasize the relationships, networks, organizations, and policies that invite participation from multiple stakeholders and remain committed to community-based and communitydriven organizing. This kind of communication infrastructure draws from some of the core concepts related to Communication Infrastructure Theory, but perhaps more critically, we rely on key extensions of the term as it relates to Culture-CenteredApproaches to Communication (CCA) and similar advancements that emphasize community voices (Dutta, 2012; LeGreco and Douglas, 2021).

\section{Communication Infrastructure for Community-Based Interventions}

Communication infrastructure frequently emphasizes various material and social resources, policies, and practices through which communities and institutions can mobilize responses to uncertainty and risk, as well as community-identified needs for change and equity. The creation and ownership of communication infrastructure can be facilitated through neighborhood storytelling networks (Kim and Ball-Rokeach, 2006; see also; Dutta, 2012; Dutta et al., 2013; Dutta and Thaker, 2019; Wilkin et al., 2010; Wilkin, 2013), community organizations and local planning committees (Heath et al., 2002), hyperlocal and community-owned media (Dutta; LeGreco et al., 2015; Wilkin, 2013). Examples of communication infrastructure frequently include local stories and spaces for community dialogue, as well as media technologies that enable community conversations and engagement.

Communication Infrastructure Theory (CIT) suggests that communities must create the social fabric that keeps individuals connected (Kim and Ball-Rokeach, 2006). These connections are not simply given, rather we build relationships through sharing stories, engaging in social interactions, collectively building policies, and organizing in ways that decenter power and instead center culture and community voices (Dutta and de Souza, 2008; Dutta, 2012; Dutta et al., 2013). In this way, the creation of a densely-connected communication infrastructure establishes a foundation for civic engagement and social activism, which can help community members prepare for when they must work together-across neighborhood and organizations-to address collective health crises, like the pandemic spread of COVID-19.

Communication infrastructure enables and constrains collective efficacy in communities, which frequently operates through four dimensions-a perceived willingness to intervene, local political control, a mix of community and instrumental support, and shared organizational participation (Sampson et al., 1999; Kim and BallRokeach, 2006). Communication infrastructure includes identifying and strengthening storytelling networks among neighborhoods. These networks involve not only narratives about personal experiences, but also a wide range of communication strategies used to construct the story of a community-including sharing stories with the media, policy advocacy, healthy eating campaigns, community celebrations, and documenting neighborhood events and activities. For example, in her review of applications of CIT, Wilkin (2013) highlighted the ways in which neighborhood-level influences, like a lack of access to healthier food options in low-income neighborhoods, can reproduce structurally-constituted health disparities. Communities with strong storytelling networks and a high sense of collective efficacy have a more developed capacity to mobilize both material and human resources to address these neighborhood-level disparities. A key piece of the efficacy surrounding these storytelling networks, however, is their sustained organizing through residents and their social networks, community organizations, and hyperlocal media. This notion of sustained organizing is explored further through more culturecentered approaches to building communication infrastructure.

Culture-centered approaches, community-based and community-driven organizing, narratives, and communication technologies are all necessarily related to communication infrastructure (Kim and Ball-Rokeach, 2006; Harter et al., 2017; Dutta and Thaker, 2019; Dutta et al., 2020; LeGreco and 
Douglas, 2021). For example, the Culture-Centered Approach (CCA) to communication (Dutta, 2012), and more recent extensions of this approach to pandemic communication (Dutta et al., 2020), emphasize the centrality of culture and community in designing health interventions. Centering culture in health interventions "anchors communicative responses to pandemics in community voices, constituted in the work of everyday organizing" (Dutta et al., 2020, p.2), but it does so in a way that prioritizes accountability to the community. In doing so, CCA frequently support radical forms of organizing that rejects neo-liberal efforts to co-opt as opposed to center community voices.

Perhaps the most essential concept provided by the CCA to our analysis of local food organizing amidst changes related to COVID-19 is the concept of infrastructures of listening (Dutta et al., 2013; Dutta, 2018; Dutta and Thaker, 2019). Infrastructures of listening create space for people to share stories and experiences from perspectives that are often relegated to the margins. By regularly and engaging these stories, requiring the voices who are routinely centered to decenter themselves and listen, and doing so in ways that are necessarily facilitated by infrastructure, communities can work across a mix of community and instrumental support to develop messages and interventions that are more meaningful during rapid responses to pandemics and other uncertain, insecure, risky, or critical health situations. At the same time, neighborhood-level interventions and research partnerships that adopt culture-centered approaches caution against performative listening, in which storytelling networks and can create the illusion of participation from the community, while reproducing power relationships that re-center the interests of more dominant voices (Dutta, 2018; Dutta and Thaker, 2019).

Second only to infrastructures of listening, we also argue that existing networks and partnerships are vital components of communication infrastructure. Sustained partnerships between universities and communities, non-profit and civic organizations, local governments, and neighborhood-level leadership are important when it comes to creating the social fabric that is necessary for collective action (Jovanovic et al., 2015). For example, in their study of communication infrastructure within the context of risk communication, Heath et al., (2002), compared both local emergency planning committees and community advisory committees, only to find that neither structure had a meaningful influence on their communities' practices and awareness of risks. Rather, the structures required a more active and routine engagement with individuals and communities in order to prompt action at the neighborhood level. Building on strong partnerships reinforces the need for reciprocity, or the mutual benefits that partners experience by participating in the sometimes arduous and often exhausting process of designing health messages and interventions that can organize and reorganize everyday practices-like figuring out how to adapting eating practices during the food shortages, store closings, and other changes during pandemic stay-at-home orders.

These infrastructures of listening and existing networks, alongside related dimensions of CIT-like the mix of community and instrumental support-can be crucial to the design of public health and food systems interventions, especially those that invite participation from multiple individuals and organizations across a community. Communication infrastructure does not emerge overnight, and when communities must coordinate actions and work collectively during periods of uncertainty and crisis-such as a pandemic_community members must act quickly and decisively to enact the shared resources required to change health practices. We continue to examine this need for communication infrastructure in the context of local food organizing.

\section{Local Food Organizing as a Form of Communication Infrastructure}

Communication practices and structures related to food security-and related concepts of food insecurity, food justice, and food sovereignty-have generated an important conversation among health, risk, and organizational communication scholars (Schraedley et al., 2020; see also; Pine and de Souza, 2013; Dutta et al., 2015; Okamoto, 2016; LeGreco and Douglas, 2017; Dougherty et al., 2018; Gordon and Hunt, 2019; Dutta et al., 2020; Ivancic, 2020). With an emphasis on local storytelling, decentralized power, environmental and economic justice, community engagement, and creating shared resources, this compelling body of research interrogates current food systems, policies, and practices and asks what we can organize together, as communities, to ensure equity and reduce disparities across our food systems. Perhaps most notably, this body of research has centered practices of creating communication infrastructure as necessary components of health communication that addresses food security.

Local food organizing to ensure equity adds a layer of complexity to this conversation by returning to the idea that even in a pandemic, people still have to eat. Changes to everyday eating practices during COVID-19 stay-at-home orders and social distancing practices have meant that consumers navigated changes in how they shopped for, ordered, and purchased food alongside the recommendations to wear a mask, socially distance, and avoid contact with large crowds. Additionally, food providers, distributors, and retailers have changed how they get food to people, including the implementation of mask requirements, new signage to control traffic flow in grocery stores and at farmers markets, increased food handling safety, and drive-thru food distribution. These changes require creative and advanced communication interventions that make it feasible for people to secure food during an insecure and uncertain situation.

Building communication infrastructure involves not only dialogue and deliberation about the ideas, problems, and solutions within a community, but also strategic efforts to coordinate groups and individuals who often hold different priorities (Sandy and Holland, 2006; Bloomgarden and O'Meara, 2007). Engaging in this kind of work through local food organizing frequently involves evidence-based strategies across short, medium, and long-term community engagement in local food systems. Community-based communication infrastructure, especially in the context of securing food during 
a pandemic, might focus the short-term organizing and likely includes documenting available resources, counseling communities on maximizing those resources; identifying quality and quantity inequities across neighborhoods; educating consumers about food resources (McCullum et al., 2005).

These strategies and conversations for building communication infrastructure through local food organizing emphasize the centrality of communication and community voices in organizing food responses during any periods of food insecurity. Moreover, they create the necessary conditions for individuals and communities to engage with institutions in complex dialogues, policy conversations, and critical reflection on topics like food systems reform (Gordon and Hunt, 2019). In other words, communication infrastructure as it relates to local food organizing can aid communities and researchers as they address food security and related questions of accessibility and affordability, food justice and questions of equity and power, and food sovereignty and the abilities of communities to build their own food systems and practices. Simply including community voices and supporting community-organized efforts, however, does not always ensure that food systems will magically become secure; that puts an impossible amount of pressure on community members to have the resources and capacity to deliver highly nuanced solutions to increasingly difficult problems. Because working with food systems means working with such a diversity of stakeholders, local food organizing has come to rely on the creation of communication infrastructure to manage the design, implementation, and evaluation of public health messages and environmental interventions aimed at food security, food justice, and/or food sovereignty. Researchers are increasingly called to align their work with a variety of communities and partners to facilitate discussions, implement and evaluate interventions, organize and manage institutional memory, and work with partners to create sustainable, community-driven action.

When applied to the specific context of organizing and reorganizing local food systems and practices as a response to COVID-19, that means some of these conversations must already be mobilized. But with an intentional and well-developed communication infrastructure with a goal of food security, communities have an opportunity to leverage that infrastructure into more nuanced and perhaps effective methods for designing public health messages and interventions. Such forms of organizing can help communities navigate periods of food insecurity-like those related to COVID19 stay-at-home order and social distancing practices.

To further consider the centrality of communication infrastructure in public health and food systems responses to COVID-19, we pose the following research questions:

RQ1: In what ways can communication infrastructure provide necessary resources for communities to respond and reorganize during periods of crisis and uncertainty?

RQ2: How can long-term investments in communication infrastructure aid communities in reorganizing local food systems during a global pandemic?

RQ 3: In what ways can communication infrastructure help communities focus conversations during the initial onset of a crisis or period of uncertainty?

\section{RESEARCH METHODS: THREE SHORT CASES OF SECURING FOOD AMIDST A CRISIS}

Our case study approach takes us to Greensboro, NC-a medium-sized city in the southeastern United States. With a population of approximately 270,000 and a metropolitan area of just over 5,00,000, Greensboro sits within Guilford County, which is home to a racially-diverse set of communities and one of the largest immigrant and refugee populations in the state. On March 10, 2020, North Carolina Governor Roy Cooper declared a state of emergency in response to the spread of COVID-19, and local stakeholders began organizing in earnest to identify health resources in Greensboro (Exec. Order No. 116, 2020). Shortly after, on March 14, North Carolina closed all public schools and prohibited gatherings of more than 100 people (Exec. Order No. 117, 2020). Stay-at-home orders were announced on March 27, 2020 through an executive order signed by Governor Cooper (Exec. Order No. 121, 2020), with the support of the North Carolina Department of Health and Human Services (NCDHHS). North Carolina then moved into a phased reopening process on May 5, 2020 (Exec. Order No. 138, 2020), with the state transitioning to Safer at Home recommendation and Phase 2 on May 20 (Exec. Order No. 141, 2020) and Phase 3 on September 30 (Exec. Order No. 169 , 2020). Modified stay-at-home orders were lifted on February 24, 2021 (Exec. Order No. 195, 2021), and the state moved to a new model of easing restrictions for gathering in public, which included restrictions related to wearing masks in public, customer capacities for businesses, and general practices for social distancing. This timeline for stay-at-home and social distancing orders also shaped how individuals and families secured food, as these orders also identified grocery stores and farmers markets as essential services, closed restaurants initially and reopened them at reduced capacity under Phase 2, and required people to adjust-or in some cases completely change-their eating and shopping habits as most meals moved to the home.

To sufficiently frame Greensboro as an appropriate site to consider community-based responses and public health messaging and interventions related to COVID-19, we must take readers back to 2009, when food access and food insecurity was first identified by the Guilford County Department of Health and Human Services (GCDHHS) as a public health concern in Greensboro. As part of their Community Health Assessment (CHA) process, the county's epidemiologist first highlighted health disparities in food access-namely limited access to grocery stores alongside high rates of heart disease and diabetes-among low-income neighborhoods in Greensboro. In what the US Department of Agriculture (USDA) would later call "food deserts," the county's epidemiologist worked alongside community and university partners to start conversations with local residents about the kinds of resources they wanted to create in their neighborhoods. These conversations would later inform his 2010 CHA report (Smith and Mrosla, 2010), and food access remained a priority in subsequent CHA reports for 2013 and 2016 (Smith and Mrosla, 2013, Smith and Mrosla, 2016). 
Amidst these local conversations about food access and insecurity, Greensboro would also make a climb on the Food Research and Action Center's (FRAC) list of major metropolitan cities experiencing food hardship - a term FRAC defines as very similar to food insecurity and focuses on poverty and access. After making local headlines when it reached the \#4 spot in 2012 (FRAC, 2012), Greensboro topped the list in 2015 (FRAC, 2015). During this time, local food organizers across Greensboro would institute several practices to help build communication infrastructure that could be leveraged into community-based and community-driven food resources. For example, in 2012, the first author worked with several non-profit groups and Guilford County health and agriculture agencies to organize a two-part community forum called Food for Thought. Hosted at the Interactive Resource Center, a day center serving Greensboro's homeless community, this forum brought together leaders from local government, individuals and organizations involved in health advocacy, non-profit and faith-based groups, and everyday community members to imagine what was possible in when it came to our local food system. Out of these conversations grew a series of local interventions and sustained communication infrastructure, some of which played vital roles in Greensboro's messaging and interventions around food access, and some of which we will discuss later in the case studies. This type of communication infrastructure created some necessary social fabric for local food organizers to begin increasing food access in Greensboro and improve our FRAC rankings to ninth in 2016 (FRAC, 2016) and \#14 in 2018 (FRAC, 2018).

In many ways, COVID-19 has tested the communication infrastructure that we have been building around food in Greensboro since 2009. So that when COVID-19 cases started to increase in the U.S., stakeholders in Greensboro were able to organize resources quickly, network numerous partners to manage our local food systems, implement local interventions to help individuals and communities adjust their eating and shopping practices, and do so in ways that prioritized food access. The short cases featured in the remainder of this essay highlight how multiple stakeholders drew upon communication infrastructure around local food organizing to assist both providers and consumers in adjusting food access, eating and shopping habits, and distribution practices in response to COVID-19. Before we examine some of the communication strategies and local food interventions that constitute these case studies, we offer a quick framing of our data collection and analysis.

\section{Data Collection}

This essay is part of a much larger and ongoing effort to examine changes in local food organizing and food security as part of the National Communication Association's Center for Communication, Community Collaboration, and Change (NCA Center). The authors are part of the Communication Studies Department that was selected as the inaugural program for what is designed to become a rotating center to promote the communication ethics highlighted in the Center's title. The authors of this manuscript received funding from NCA to work alongside a local farmers market initiative-the Neighborhood Markets-to focus on food access and food justice in low-income communities. As such, the qualitative case studies included here are presented as preliminary data, from both the Neighborhood Markets project and other community-based efforts, that document some initial and immediate observations that we considered relevant to food systems communication amid compounding crises.

Data collection for this project is rooted in multiple, related qualitative and community-based research projects related to food security and food justice in Greensboro, NC. The first author has been immersed in local food organizing from a community-based perspective since 2009, when she began partnering with the Guilford County Department, several nonprofit organizations that focus on food access, and numerous neighborhoods with low food access and high rates of poverty. As such, she has a long history of working across communities to build the kinds of communication infrastructure that are needed during the reorganization of food systems during a pandemic. Data collection also included participation from a larger research team, which included the second and third authors, as well as a research partnership with market managers and farmers from the Neighborhood Markets project, although the latter group's participation focused exclusively on the third case study featured in this essay. In particular, the second and third authors were funded through the NCA Center to provide research support, including survey construction and on-site interviews with vendors and customers, as well as technical support, including content production and website development for the Neighborhood Market partners.

The short case study examples included in this essay are informed by the following qualitative sources of data:

- The first author's participation in a series of public meetings hosted by Guilford County Schools and a local foundation to enact a school meal network for students who were now staying at home. Meetings were hosted weekly, starting in March and moved to bi-weekly or monthly starting when North Carolina moved into Phase 2 on May 22. The first author attended four meetings between March 16 and May 22 , and the research team collected meeting minutes and zoom recordings for the remaining meetings that have been made publicly available.

- The first author's coordination of multiple stakeholder and community members in the creation of a Greater Greensboro Food Resources guide, which directed people who were now required to stay home to available food resources that individuals could obtain through limited or no contact and within local and state policy guidelines. The first author kept detailed field notes and email exchanges to reconstruct the case study examples, and the research team track and monitored guide use through both bitly.com and Google analytics.

- The research team's documentation of a community-based partnership with the Neighborhood Markets, a collaboration between two farmers markets-the Corners Farmers Market and the People's Market in Greensboro-who are working to promote equitable food access through neighborhood-based 
food markets. The first author began working with the Neighborhood Markets to help develop a sustainability plan for their Green4Greens program, which is designed to double the dollars for customers who use SNAP/EBT to purchase food items at either market. That work transformed into an effort to keep the Corner Farmers Market open during COVID-19 stay-at-home orders. This case includes 25 individuals interviews that were conducted through faceto-face, online, and phone conversations, as well as 55 online surveys. Both the survey and the interviews included questions to assess how/if participants' eating and shopping habits have changed, as well as how the market has responded to customer and vendor needs to access food.

\section{Data Analysis}

Considering that this project is ongoing, our data analysis continues to develop as we work alongside our research partners. At the same time, the authors were able to isolate some initial insights that focus on the design and implementation of public health and local food interventions and demonstrate the creative products of an intentional communication infrastructure. Our analysis draws from iterative and constant-comparative methods of analyzing qualitative data from numerous sources (Strauss and Corbin, 1997; Tracy, 2019), as well as discourse tracing methods of establishing timelines to reconstruct case studies (LeGreco and Tracy, 2009). We engaged in both open and axial coding to make connections between existing communication infrastructure and the emergence of new needs related to securing food during stayat-home orders. We also focused on documenting processes, as well as integrating tracking and monitoring data to illustrate how communication played a part in local interventions around food. We refined our observations to identify the core pieces of each narrative and provide useful insights for engaged food systems communication scholars and organizers.

\section{FOOD AND COMMUNICATION INFRASTRUCTURE IN RESPONSE TO COVID-19}

When stay-at-home orders were implemented in North Carolina on March 27, 2020, local food stakeholders-including grassroots organizers, local businesses, and city and county health agencies-had already been partnering to reorganize food resources and create new mechanisms to access and distribute food. The following three examples illustrate some of the key features of communication infrastructure that enabled strategic interventions and related public health messaging to help individuals and families find food as COVID-19 cases began to spread. We focus specifically on the time period between March 27 and May 2, which encompasses the initial stay-athome and social distancing orders through the Phase 2 reopenings. We also offer supplemental stories and examples that continued through October 2, when NC moved into Phase 3 reopenings and additional easing of restrictions. In doing so, we pay particular attention to how communities responded and reorganized during that initial onset of uncertainty and reorganizing during the pandemic. Each case study speaks to each of the three research questions on some level. The first case study emphasizes existing communication infrastructure around school meal programs as its central feature, while the second case highlights how the collective documentation of resources serves a sustainable way to aid communities and community organizations in finding food resources, and the third case study focuses on the ways that a grassroots farmers market community engaged its customers and vendors to navigate the initial period of uncertainty. Across these cases is an attempt to identify the communication infrastructures that individuals, communities, and institutions turned to initially during those early phases of COVID-19 reorganizing.

\section{Securing School Meals Through Community-Based Infrastructure}

On March 14, 2020, Guilford County Schools (GCS) announced that public schools would be closed, likely through the end of the 2019-2020 the school year, and remaining instruction would move online. Within 3 days of this announcement-on March 17-GCS launched a 33-site school meal network, which allowed the school system to provide supplemental school meal programs and additional food resources for students who would now be learning from home. As part of this school meal network, any person who was 18 years old or younger, regardless of their enrollment status at GCS, could pick up a grab and go meal between 11:30 am and 12:30 pm, Monday through Friday. Food was provided through the USDA's National School Lunch Program (NSLP) in partnership with GCS, and pickup locations included local recreation centers, faith-based organizations, and existing food pantry drop sites.

The implementation of the school meal network, particularly the speed with which GCS was able to launch a highlycoordinated, 33-site intervention, can be traced in part to community-based communication infrastructure and existing food networks that could support such an endeavor. The GCS school meal network was modeled after Greensboro's summer meal network-a grassroots effort launched in 2015 in partnership with the City of Greensboro's Community Food Task Force-which provided supplemental meals during the month of August. During June and July, the NSLP provided access to food directly through the school systems; however, August was considered a "gap month," in that the NSLP did not provide food to students-some of whom were dependent on school meals for daily access to food. To fill that "gap month," local food organizers had created a densely-connected network of food pantries, faith-based organizations, recreation centers, and other neighborhood partners to ensure access to meals for students when the NSLP could not. With support from the city's Community Food Task Force, the summer meal network had already been effectively filling food gaps for students for 5 years before COVID-19 disrupted food access during the 2019-2020 school year.

Both the summer meal network and the GCS school meal network that was modeled after it in response to COVID-19 can 
be traced even further back to the 2012 Food for Thought event that we first mentioned in the previous section. The two-part event started with a community viewing of the film A Place at the Table, followed by presentations from Guilford County's Department of Social Services (GCDSS) and several non-profit and community-based organizers about the landscape of food access and SNAP/EBT usage in Greensboro. Two weeks later, participants were invited back for part two, which featured several breakout groups that asked people to imagine the food resources and communities they would like to build in their neighborhoods. Out of these small-group discussions grew several interventions that contributed to Greensboro's communication infrastructure around food, like mobile farmers markets and local food policy councils. One of the breakout sessions focused on providing meals for K-12 students to fill the "gap month" when the NSLP did not operate. That session and the momentum generated through the Food for Thought event would inspire service groups-like a local Greensboro chapter of Rotary International-to spark conversations with food pantries and backpack programs, neighborhood leaders, representatives from the City's Parks and Recreation Department, and other food stakeholders to create the summer meal network.

As such, when the increasing cases of COVID-19 forced the closure of schools in Guilford County in March 2020, GCS was not completely unprepared to manage local food needs for their students. They simply tapped into the existing relationships, networks, and communication infrastructure that had been established to fill previous gaps in food access for students. In doing so, GCS also enacted an intervention and a structure with which many students were already familiar, as some of them were already accustomed to visiting the grab and go distribution sites during the summer months. Thus, community-based organizing and communication infrastructure played a key role in GCS's quick response to school closings related to COVID-19, because they provided a foundation from which local partners could quickly develop interventions to ensure food access for students.

Moreover, these existing networks reinforce the importance of sustained communication, storytelling, and world-building (Jovanovic et al., 2015) in the service of securing food during periods of insecurity. Threads of the school meal network connect back to conversations that were started through communitybased dialogues and opportunities for neighborhood-driven storytelling 8 years before the network was needed during the COVID-19 pandemic. This kind of sustained communication can help communities build the kinds of infrastructure that become useful over time for a variety of stakeholders.

\section{Coordinating Food Access Through Documenting Resources}

The dialogues and interventions that were initiated around food access for K-12 students also created the conditions to amplify other food resources that were available during COVID-19 social distancing and stay-at-home orders. At one of the weekly, public meetings hosted by GCS in March 2020, the first author was asked to speak about additional efforts to organize food resources, especially considering that increasing numbers of Greensboro residents were left without work due to COVID-19 closings. Combined with short-term food shortages, due primarily to overbuying and hoarding at the start of the pandemic, the reduction of income faced by many individuals and families meant that some would struggle to find food and most residents would need to adjust their shopping and eating habits in at least some way. During that meeting, the first author challenged partners to begin working on a coordinated list of local food resources that could be made available to Greensboro residents in a format that was both easily shareable and updateable. As she mentioned to the group, "you all are working so hard to create and coordinate some very useful resources, but if no one knows about them, no one is going to use them."

From that meeting in mid-March, a small group of local food organizers branched off from the K-12 schools conversation to begin assembling the Greater Greensboro Food Resources guide, as well as a similar guide for neighboring High Point. With UNCGreensboro's Lifetime Eating and Physical Activity Program (LEAP) and the Greater High Point Food Alliance coordinating the conversation, organizers created a Google Doc that could direct people to food resources in their respective communities. Organizers settled on a Google Doc as a method of distributing information, because we could create a document that linked users directly to the organizations and communities providing the resources, and we could do so using a platform that was easily updateable and shareable across websites, social media pages, and email listservs. The document centered communication infrastructure, specifically the creation of communication resources that could be shared across a variety of media, as a mechanism for disseminating public health messages related to securing food during the spread of COVID-19.

The Greater Greensboro and Greater High Point Food Resources featured a dashboard that helped us streamline how users could access information that was most relevant for their needs. The dashboard featured six components-food assistance, grocery store information, local farms and farmers markets, local restaurants for takeout and delivery, volunteer, and donate. Within each link from the dashboard, users were directed to a collection of resources and information that had been sourced by local food organizations and community members. For example, under "Food Assistance," users could find links to the GCS school meal network and other food support for K-12 students. They could also find information about food pantry locations and drive-up free meals being offered to community members through local non-profit organizations. Under "Grocery Store Information," the resources guide provided updated information about grocery store hours, special hours for seniors, and mask policies before statewide mask ordinances were instituted. Through the "Local Restaurants for Takeout \& Delivery" links, users were taken to websites and social media pages that had been organized by neighborhood associations to identify restaurants that remained opened, albeit in a different capacity. Users could also access social media pages that documented people's experiences finding food in the midst of a pandemic, like the Greensboro Takeout Facebook page-a site started by a city 
councilwoman to share information about local restaurants providing curbside, carryout, and delivery options-as well as the GreensboroBLACK Food Mob-a site started specifically to promote Black-owned restaurants and food businesses who offered those same services.

Local food organizers maintained the Google Doc from March 20 through May 22, primarily as a resource for local residents as they transitioned through stay-at-home orders. During the active use of these resource guides, we accounted for over 2,490 unique views of the Greater Greensboro document and 750 unique views of the High Point document. Document views were concentrated most highly during the first 2 weeks after stay-at-home orders were announced, with use slowly tapering off as Greensboro approached Phase 1 in early May. We suspended updates when Greensboro moved into Phase 2 reopening, and an archive of the Greater Greensboro Food Resources guide remains available at bit.ly/GSOfoodDoc.

The case of the Greater Greensboro and Greater High Point Food Resources guides demonstrated how local food organizers created the conditions for building new and expanding the existing communication infrastructure around food in Greensboro. These coordinated resources proved useful in numerous situations, such as when a single mother of two young children contacted the first author via email about finding food. She was laid off from her job due to COVID-19 and she had recently qualified for the Women, Infants, and Children (WIC) food program; however, her benefits had not yet come through. Due to food shortages at her usual grocery stores, she was having difficulty finding staple items like milk and other food to feed her family. The first author was able to use the Food Resources guide to help her find food pantry drop sites that were providing the food she needed that day, as well as free meal locations to help her find food for the remaining week. Additionally, the guides have carved out space to continue generating resources for communication infrastructure that are owned by and accountable to the community, such as the GreensboroBLACK Food Mob Facebook page. The page has remained active throughout stay-at-home orders and phased reopening, even planning events to support Black-owned restaurants throughout the pandemic.

\section{Creatively Reorganizing Food Access Through an Infrastructure of Listening}

Our final case example focuses on the creative reorganization of food access in partnership with the Neighborhood Markets, specifically the Corner Farmers Market in Greensboro. The Corner Market is a grassroots-organized neighborhood market that sits on the border of a middle-income neighborhood, and a neighborhood that the US Department of Agriculture defines as both low-access and low-income (USDA, 2021). The Corner Market was built on a philosophy of creating neighborhoodbased food markets that are driven by residents of the communities and geographic areas relative to the market(s). Although some vendors and one of the anchor farms for the Corner Market reside outside the neighborhood, the majority of vendors, organizers, and market managers live within a mile of the market. The market was established in 2013, and in 2018 began partnering with a fledgling market two neighborhoods across town-the People's Market. They formed the Neighborhood Markets, an umbrella organization that would allow the two markets to share resources, like SNAP/EBT accounts, and provide mutual supports in reaching their customers and vendors.

During a March 9, 2020 meeting of the Neighborhood Markets research team, which included the first author and two of her graduate students, as well as representative from both the Corner Market and the People's Market, the weekly conversation started with plans to develop a website for their combined Green4Greens program. The partnership-including the three members of the research team, as well as our research partners from the community-was funded by NCA's Center for Communication, Community Collaboration, and Change to create a sustainable funding network to double the dollars for SNAP/EBT users at both markets. Early in that March 9 conversation, however, the market manager for the Corner Market brought up rumors that Governor Cooper would declare a state of emergency regarding COVID-19, perhaps as early as that afternoon (the declaration would come the next day). She was concerned about the team's ability to keep the Corner Market open, as well as to open the People's Market for the season. ${ }^{1}$ The members of the research team paused the meeting to consider that we might need to focus less on Green4Greens and, at the present moment, concentrate more on how the markets can respond and intervene as communities faced the spread of COVID-19.

Shortly after the state of emergency was declared in North Carolina on March 10, the market manager contacted our state representative to confirm that farmers markets were considered essential space for food access-similar to grocery stores-and would not be subject to closing their doors. The Neighborhood Market partners could then focus on how to reorganize the Corner Farmers Market to respond to COVID-19. We considered options like creating an advance ordering and drive-thru pickup model to keep the market open while promoting social distancing, as well as outlining conditions for when we would close the market, like if the market manager developed symptoms of the virus. But before we chose to implement any of those ideas, we planned to host two listening sessions at the upcoming Corner Market on March 14.

The first listening session was held during the market, primarily for customers, with the first author and an organizer for the People's Market sitting at a market booth with a sandwich board sign that read "Times are Weird, Let's Chat." 18 customers participated in the listening session across the three-hour market. In an effort to adopt social distancing practices, most of the conversations occurred interpersonally (as opposed to in small groups) between the market organizers/research team and the customers. Participants frequently started their conversations

${ }^{1}$ The Corner Market operates as a year-round market on Saturday mornings, while the People's Market is a seasonal market that operates from April through October on Thursday evenings 
with questions, seeking what information market organizers had about the virus and its potential impact on our food system. As one neighborhood resident posited, "you don't think you're going to have to close, do you? One thing you have going for you is that you're outdoors, and I've heard the virus hates sunlight!!” At least two conversations started with attempts to figure out what sixfoot social distancing looked like, as people were still becoming accustomed to staying further apart. When pressed on what the markets should be doing, one participant said, "keep doing what you're doing-maybe spread out a little more-until somebody makes you stop," while another said simply "keep the market open as long as possible."

Perhaps these initial comments are not surprising, as we spoke primarily with people who were still shopping at the market even after a state of emergency had been declared for NC; however, these insights were also echoed in the interviews, almost all of which occurred off-site from the market. In these subsequent interviews with Corner Market customers, specifically regarding their concerns about finding food going into stay-at-home orders, one neighborhood resident stated, "it makes me feel better to know that the market is there. That I can get food just down the street. Going into summer, it makes me feel safer to know that I can still get fruits and vegetables that have-quite frankly-passed through fewer hands to get to me." While the listening sessions and interviews gave market organizers confidence that customers would still support the market amidst the growing pandemic, and gave us an opportunity to discuss additional details like the advanced-ordering and drivethru pick up model, they also gave organizers a clearer understanding of how meaningful a resource the Corner Market had become to the neighborhood. One that residents did not want to lose as restaurants began closing and grocery stores began limiting their hours.

The second listening session was held immediately after the market, on the back porch of one of the anchor farmers, who lived just down the street. Although this meeting was held with vendors, several of the people on the porch also lived in the neighborhood, were customers at the market, and at least one of them used the SNAP/EBT program. Our goal was to assess the community's capacity for continuing to operate, as well as the interests in developing an advanced-ordering and drive-thru pickup model. We reported the quick results from the listening session with the customers, including the interests in developing the advanced-ordering model. The vendors highlighted some of the health practices that they had already implemented, but how some of those practices would not be sustainable over time. As one vendor mentioned, "we brought 100 pairs of disposable gloves, and we went through all of them. We changed gloves for every transaction, and I'm not sure that's something we can afford to keep doing on our own. So having the advanced orders might be really good for us."

The group talked through additional practices, such as providing hand sanitizers, and the likelihood that organizers, vendors, and customers would have to begin wearing masks in the near future. When it came time to make some final calls about keeping the market open and developing a drive-thru, one of the anchor farmers spoke up and said, "whatever you need. We'll figure it out," and all of the participants agreed. These sessions, with both customers and vendors, introduced the potential for infrastructures of listening (Dutta, 2018; Dutta and Thaker, 2019) as a communication practice that could help the Neighborhood Markets design interventions that could accomplish the goals as outlined by the participants.

The Neighborhood Market partners quickly collaborated to design and implement the advanced-ordering and drive-thru pickup model at the Corner Farmers Market, as suggested by partners at the March 9 meeting and later supported by both the customers and vendors at the listening sessions on March 14. The market manager added a webpage to the Corner Market site that linked customers directly to contact information for individual vendors who were participating. Customers were instructed to contact vendors to order and pay for their items directly. On Saturday mornings, vendors dropped off labeled items at the drive-thru tent, which was located in the parking lot of an adjacent restaurant that remained closed due to COVID-19. Customers had options to walk up or drive through and pick up their orders. Market volunteers also offered limited contact interactions, and they would load purchases into the cars as each customer moved through the line.

This model worked well for customers who were used to ordering online for food, or who could pay ahead directly to the vendor. However, SNAP/EBT users were required through an agreement with the USDA to run their transactions through the market manager; therefore, we created a space for SNAP/EBT users to pay the market manager on pickup, and SNAP/EBT users were the only group of customers who were not required to pay in advance. Once the market manager figured out how to include SNAP/EBT users in the reorganization of the Corner Market, partners began to track participation for the advanced-ordering and drive-thru, as well as well general SNAP/EBT dollars spent at the market.

The advanced-ordering and drive-thru pickup model was launched on May 20 and qualified as an overwhelming success, especially during the period of time between March 27 and May 8, when full stay-at-home orders were in effect. At the height of its usage, 375 advanced orders were fulfilled in a 3-h period, and that level of participation was sustained throughout most of the month of April and parts of May 2020. The research team continued to track advanced orders through November 21, 2020, which is when the Corner Market moved to a more sophisticated system for centralized online ordering and adopted a new method for tracking orders. During this initial time period, the Corner Market fulfilled 5,893 advanced orders. Figure 1 shows the relationship between use of the advanced-ordering system and the timeline for stay-athome and phased reopening through Phase 2.

The trends clearly document a relationship between the volume of orders and the various stages of stay-at-home orders and phased reopening. Simple frequency counts demonstrate how advanced order numbers peaked during stay-at-home and fell steadily as Greensboro moved out of Phase 1 and into Phase 2. These observations were also affirmed in the interview and survey data. Of the 55 survey participants, 25 of them identified using the drive-thru pickup 


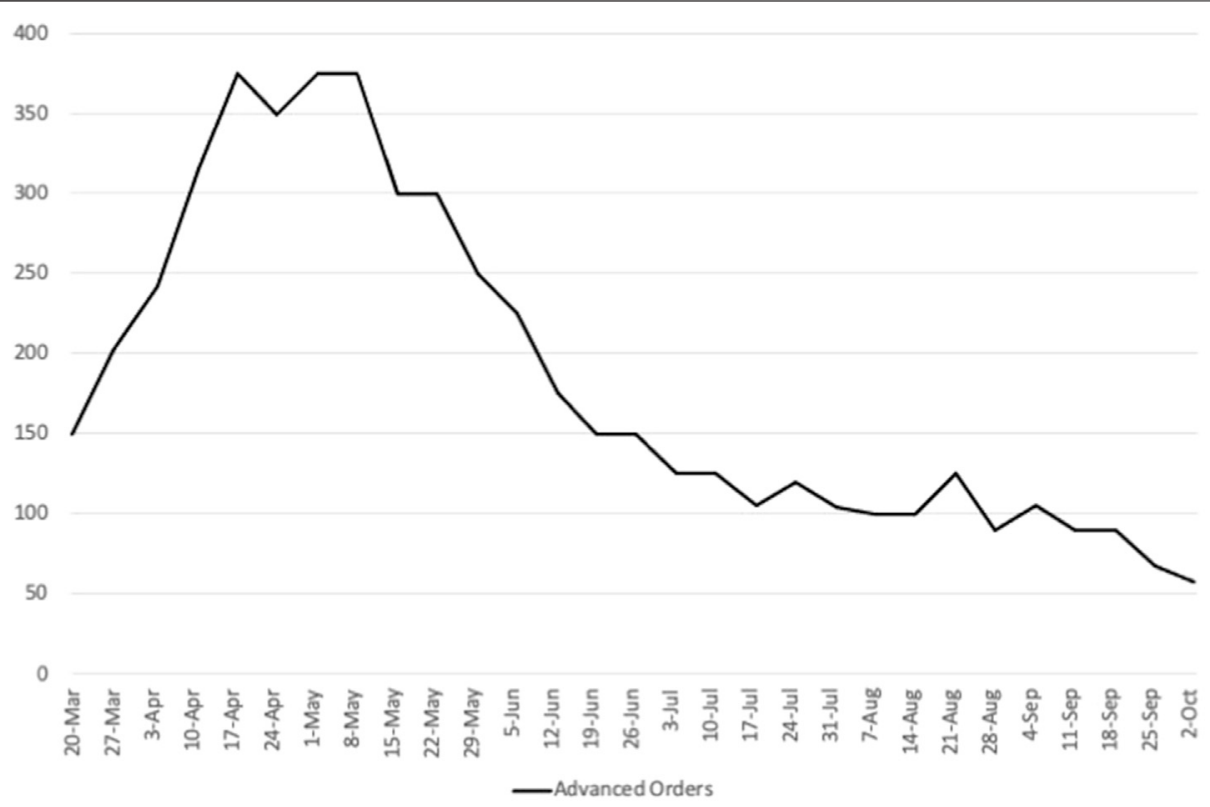

Stay-at-home Phase 1

Phase 2

FIGURE 1 | Advanced orders at the corner farmers market.

system as a method for finding food during stay-at-home and social distancing orders. As one participant noted, "The drivethru was especially important in the very beginning, when you couldn't get a slot at the [regional grocery store] for drive-thru pickup." Another survey participant highlighted how the advanced ordering helped them navigate uncertainty around new risks to their family, stating "we love it!! We have a high risk family member so this makes it possible for us to continue buying goods from the market. If it wasn't available we would not shop at the market." The trends suggest that customers were most reliant on the advanced orders and drive-thru pickup when the local food system was in the greatest flux.

The infrastructures of listening instituted at the Corner Farmers Market allowed for a more nuanced approach to reorganizing access at the market. These practices were beneficial not only for drive-thru customers, but also for SNAP/EBT customers. In a single Saturday in July 2020, the Corner Market doubled more than $\$ 700$ in SNAP, which was equivalent to what they had doubled in the entire month of July in 2019. SNAP/EBT users noted in personal interviews that the doubling program at the Corner Market helped stretch their food dollars during COVID-19 and as one mom of a special needs child noted, "the program has been a gamechanger, because I'm able to get access to many of the gluten-free foods I need for my daughter." She spoke about noticing an immediate change in the supply chain when it came to gluten-free products, with some grocery stores having limited or no access when products sold out. The benefits provided to SNAP/EBT customers at the Corner Market were also illustrated in the survey data. Although only five of the 55 survey participants reported using SNAP/EBT, four of the five reported facing barriers to finding food. Their responses included "shortages of stable items at grocery stores" from one SNAP/EBT customer, "general access to food I know I can pay with SNAP," from another, and even "using food stamps" from a survey participant who had recently become SNAP-eligible after losing employment due to COVID-19.

As a response to COVID-19 stay-at-home orders and social distancing, infrastructures of listening alongside existing networks and community-based organizing made reorganizing food systems and local food practices possible at the Corner Market. Although some of the conversations gravitated toward practices that sometimes reified dominant market structures, such as the relationships between customers and vendors and the reliance on SNAP/EBT to provide food assistance, they also created a moment for the organizers and research team to begin collecting stories and building the kinds of storytelling networks and practices that can imbue grassroots markets with a more culture-centered approach. In one of the lengthier and more provocative interview related to COVID-19 reorganizing at the Neighborhood Markets, the first author spoke with someone who was a SNAP/EBT user at the Corner Market and had worked as a vendor at the People's Market. He mentioned how the efforts to reorganize practices at the markets "made everything less scary during COVID-19.” Even more, he spoke directly to how he had been able to cultivate his own sense of community through the market, stating "some of the farmers and the folks there are my friends. I met them all through what they were doing. Like, we weren't friends before the market, but now we've even worked on projects together." This participant also highlighted how grassroots markets like the Corner Market and the People's Market are a different kind of model that allows for more decentralized relationships across our food system. "For me," 
he said, "it goes beyond purchasing power when I can support the people I can talk to. There's more of an ability to thrive when things are decentralized."

\section{CONCLUSION}

The case studies presented in this essay reinforce the importance of communication infrastructure, both in the design of health and food systems interventions, as well as their application in the context of food security. Examples from school meal programs, community resources guides, and drive-thru pickup models at local farmers markets have illustrated the centrality of communication infrastructure in engaging communities and mobilizing their members to imagine new possibilities for secure food systems. These observations are in line with where both food communication scholars and communication activism scholars are pushing our discipline (e.g., Dutta and Thaker, 2019; Schraedley et al., 2020; LeGreco and Douglas, 2021). These approaches to communication scholarship and practice not only encourage us but frequently compel and even require us, as researchers, to engage directly with the individuals and communities that are involved in the contexts we study. Even going so far as to create structures of community-driven ownership and accountability in organizations and practices we help build.

Taken together, the three cases in Greensboro have clearly demonstrated how existing resources and relationships enabled quick responses when routine food practices changed almost overnight, but the networks that launched these resources and mobilized these relationships did not appear in response to COVID-19. Rather, they reflect almost 10 years of organizing, building trust, changing policies, and communicating across institutions, nonprofits, and neighborhoods (LeGreco and Douglas, 2021). In the case of the Corner Farmers Market and the People's Market, for example, the first author had worked with each of the three market managers on separate projects, independent of the markets, before she partnered with them on their Green4Greens and advance ordering system. Each Neighborhood Market partner recognized how we could shift focus from fundraising for SNAP-doubling to advocating for the market to remain open and creating a system to keep vendors and customers connected during COVID-19, because we already knew how to partner with each other from a community-based perspective. Because of this, we recognized the opportunities to enact our infrastructures of listening and reach out to our communities before COVID closings threatened to keep us apart; we tested out a creative yet practical way to keep vendors and customers connected to ensure that people still had access to food; and we refocused our attention on supporting our SNAP customers as the reorganizing unfolded. The sister markets have even witnessed an expansion in their capacity during this crisis, as they have maintained a $400 \%$ increase in SNAP usage at the markets, compared to their prepandemic seasons, and the Corner Farmers Market has upgraded to a centralized advanced ordering system as a way to permanently expand their services to include the drive-thru pickup.

At the same time, we must also remain cautious as communication researchers who make observations and help design community-driven and community-based interventions, as our work also raises important questions about power and agency, particularly from a culturecentered approach. For example, the practice of hosting listening sessions was introduced to the Neighborhood Market partners in the spirit of the infrastructures of listening as envisioned by Dutta and his various colleagues (2018; Dutta and Thaker, 2019). One aspect of these infrastructures that Dutta urges us to avoid is performative listening, where organizers create the illusion of input, but marginal voices continue to be overshadowed by stronger ties and existing relationships within the neighborhoods. Practices of regularly engaging the neighborhoods in listening sessions to identify challenges within our food systems have yet to become regular and routine across the two markets. Moreover, some of the early conversations seemed to reify dominant structures of power and agency, such as relationships between customers and vendors and the reliance on SNAP/EBT to fund community support efforts. We must also acknowledge, however, that the grassroots and pop-up nature of both the Corner Market and the People's Market gives them the opportunity to continue moving toward a more idealized version of a culture-centered approach. These two markets are very much "owned" by the people in their respective neighborhoods, with supplemental support from some farmers and vendors who are not residents. What both markets are working toward doing now is building the types of communication infrastructure that will enable them to stay committed to their neighborhood-based approach. We will continue to address this central tension as the research develops further.

One of the most challenging aspects of building communication infrastructure-particularly as a method to prepare for crisis, uncertainty, and insecurity-is that communities and partners are often creating resources and relationships that they are not yet sure how they will use. Indeed, when we first organized the aforementioned Food for Thought events in 2012, partners never once considered how the work we were doing would produce networks, relationships, and resources that would help us 1 day respond and reorganize during a pandemic. Within this space of unknown futures, however, we as communication scholars can do some of our most important work. We can assist communities and neighborhoods as they recognize the importance of communication and build the infrastructure necessary to support it. We can document processes and help partners collect data, so they can make sense of the work they are doing with their communities. Perhaps most importantly, we can use communication infrastructure to help carry the narratives that communities use to stitch together their experiences, resources, and potential into a truly resilient structure of support. 
Although the work related to these cases is ongoing, the preliminary data suggest that the communication infrastructure that we have built around food in Greensboro has indeed served the community in the design of health and food systems interventions related to COVID-19. The pandemic has represented one of the first true tests of how well our community can respond to securing food access in times of crisis and insecurity. While it would be premature-and perhaps a bit naïve-to suggest that our attention to communication infrastructure has ensured access and equity for all community members, we do argue that Greensboro is moving in the right direction when it comes to creating the types of symbolic and material moves that are required for building vibrant, resilient, and secure food systems. This observation is also evident during the pandemic, with the launch of the Grove Farmers Market in East Greensboro-another project in partnership with the NCA Center for Communication, Community Collaboration, and Change, with this market focusing on creating opportunities for Black and brown farmers.

Our analysis of three cases in Greensboro must also acknowledge some of the limitations of this study, which give rise to opportunities and calls for continued research. We chose to focus on Greensboro, because of our proximity to this community and its neighborhoods during COVID-19 closures, as well as our own embeddedness as communitybased researchers. As such, we recognize that some of our observations are indeed indicative of Greensboro and may be limited to communities and metropolitan areas similar to it. Initial reports of reorganizing food systems from comparable communities have reinforced the need to build resilient systems through multi-sector partnerships, advanced ordering and curbside food distribution, and increased attention to voices along the margins (Community Food Lab, 2020). So while some of our initial observations may transfer to communities beyond Greensboro, we also acknowledge that our analysis has privileged an urban perspective based in the southern United States. As we submit this essay, we remain in a global pandemic, and we would be remiss if we did not reminder readers that we live within not only local but global food systems. Across the multitude of layers that make up our many food systems, different resources, relationships, partnerships, and structures converge in ways that make food easier to access in some neighborhoods and households than others-especially among poor and rural communities and communities of color. As communication research that addresses food systems continues to grow, scholars are well positioned to examine how communication infrastructure both enables and constrains how communities can remain resilient in the face of immediate food crises and ongoing food insecurity.

Another methodological limitation of our case study approach concerns our emphasis on initial responses to COVID-19 stay-at-home and social distancing orders. Our approach was very intentional in that we wanted to examine how communication infrastructure could enable and constrain the food system reorganization in the first few months of what would become almost a full year of
COVID closings. In other words, our goal was to illustrate how partners worked together to coordinate and mobilize resources around local food access in the immediate reorganizing around COVID-19. What we have only begun to examine is how everyday individuals and families used those resources to weather that remaining year of the pandemic. We have some preliminary data from the Corner Farmers Market-such as the $400 \%$ increase in SNAP usage at the markets-that suggests customers have leaned heavily on local markets during the pandemic. However, we do not have access to the same tracking and monitoring data to examine how K-12 students and their families utilized the school meal network. Anecdotally, we have stories of the mother and father who walked their daughter to her nearby elementary school to pick up food every Monday through Friday, not only to get breakfast and lunch, but also as a way to add in some daily physical activity. At the same time, we also have the story of a single father of four who picked up food for his children for 1 week, but stopped after they repeatedly refused to eat the mostly packaged and heavily-processed food options. The COVID19 pandemic has given scholars the opportunity to examine not only how communities responded and reorganized food systems, but also how individuals and families reorganized their own eating practices in light of limited access to their regular food routines. Future research must continue to expand this emphasis on how individuals, families, neighborhoods, and communities use the resources that are available to them, particularly as they make decisions about something as everyday as what to eat. Even more, culturallycentered approaches that emphasize communication infrastructure can push this research further by considering how communities also participate in the construction of those resources to begin with.

As local food organizers in Greensboro continue to move closer toward culturally-centered, community-based forms of food organizing, much work remains. Key voices in these conversations continue to rely on executive and civic leadership from within non-profit and government agencies, and advocates and activists sometimes struggle to center the voices that are regularly relegated to the margins. Witnessing how local food organizers in Greensboro responded to COVID-19, however, gives us hope that working toward food security-even amidst a pandemic-is more than possible.

\section{DATA AVAILABILITY STATEMENT}

The original contributions presented in the study are included in the article/supplementary material, further inquiries can be directed to the corresponding author.

\section{ETHICS STATEMENT}

The studies involving human participants were reviewed and approved by the Institutional Review Board at the 
University of North Carolina at Greensboro. The patients/ participants provided their written informed consent to participate in this study. Written informed consent was obtained from the individual(s) for the publication of any potentially identifiable images or data included in this article.

\section{REFERENCES}

Bloomgarden, A. H., and O'Meara, K. (2007). Faculty Role Integration and Community Engagement: Harmony or Cacophony? Mich. J. Community Serv. Learn. 13, 5-18.

Community Food Lab (2020). Crisis, Opportunity, \& Resilience in NC's Local Food System: A 2020 NC Farmers Market Survey \& Action Proposal. Available at: http://communityfoodlab.org/projects-archive/fmsurvey.

Dougherty, D. S., Schraedley, M. A., Gist-Mackey, A. N., and Wickert, J. (2018). A Photovoice Study of Food (In)security, Unemployment, and the DiscursiveMaterial Dialectic. Commun. Monogr. 85, 443-466. doi:10.1080/ 03637751.2018.1500700

Dutta, M. J., Anaele, A., and Jones, C. (2013). Voices of Hunger: Addressing Health Disparities through the Culture-Centered Approach. J. Commun. 63, 159-180. doi:10.1111/jcom.12009

Dutta, M. J. (2018). Culture-centered Approach in Addressing Health Disparities: Communication Infrastructures for Subaltern Voices. Commun. Methods Measures 12, 239-259. doi:10.1080/19312458.2018.1453057

Dutta, M. J., and de Souza, R. (2008). The Past, Present, and Future of Health Development Campaigns: Reflexivity and the Critical-Cultural Approach. Health Commun. 23, 326-339. doi:10.1080/10410230802229704

Dutta, M. J., Elers, C., and Jayan, P. (2020). Culture-centered Processes of Community Organizing in COVID-19 Response: Notes from Kerala and Aotearoa, New Zealand. Front. Commun. 5, 62. doi:10.3389/fcomm.2020.00062

Dutta, M. J., Hingson, L., Anaele, A., Sen, S., and Jones, K. (2015). Narratives of Food Insecurity in Tippecanoe County, Indiana: Economic Constraints in Local Meanings of Hunger. Health Commun. 31, 647-658. doi:10.1080/ 10410236.2014.987467

Dutta, M. J. (2012). Hunger as Health: Culture-Centered Interrogations of Alternative Rationalities of Health. Commun. Monogr. 79, 366-384. doi:10.1080/03637751.2012.697632

Dutta, M. J., and Thaker, J. (2019). 'Communication Sovereignty' as Resistance: Strategies Adopted by Women Farmers amid the Agrarian Crisis in India. J. Appl. Commun. Res. 47, 24-46. doi:10.1080/00909882.2018.1547917

Exec. Order No. 116 (2020). Declaration of a State of Emergency to Coordinate Response and Protective Actions to Prevent the Spread of COVID-19. Available at: https://files.nc.gov/governor/documents/files/EO116-SOE-COVID-19.pdf.

Exec. Order No. 117 (2020). Prohibiting Mass Gatherings and Directing the Statewide Closure of K-12 Public Schools to Limit the Spread of COVID19. Available at: https://files.nc.gov/governor/documents/files/EO117-COVID19-Prohibiting-Mass-Gathering-and-K12-School-Closure.pdf.

Exec. Order No. 121 (2020). Stay at home Order and Strategic Directions for North Carolina in Response to Increasing COVID-19 Cases. Available at: https://files. nc.gov/governor/documents/files/EO121-Stay-at-Home-Order-3.pdf.

Exec. Order No. 138 (2020). Easing Restrictions on Travel, Business Operations, and Mass Gatherings: Phase 1. Available at: https:/files.nc.gov/governor/ documents/files/EO138-Phase-1.pdf.

Exec. Order No. 141 (2020). Easing Restrictions on Travel, Business Operations, and Mass Gatherings: Phase 2. Available at: https://files.nc.gov/governor/ documents/files/EO141-Phase-2.pdf.

Exec. Order No. 169 (2020). Restrictions to Protect Lives during the COVID-19 Pandemic: Phase 3. Available at: https://files.nc.gov/governor/documents/files/ EO169-Phase-3.pdf.

Exec. Order No. 195 (2021). Lifting the Modified Stay at home Order and Easing Certain Restrictions on Businesses and Gatherings. Available at: https://files.nc. gov/governor/documents/files/EO195-Easing-Restrictions.pdf.

FRAC (2012). Food Hardship in America 2011. Available at: https://www.tiki-toki. com/timeline/entry/842615/Food-Security-in-the-Piedmont-Triad/.

\section{AUTHOR CONTRIBUTIONS}

The first author was responsible for the overall framing, data collection and analysis, and writing of the article. The second and third authors were responsible for additional data collection and assistance with final writing and references.

FRAC (2015). How Hungry Is America? Available at: https://www.tiki-toki.com/ timeline/entry/842615/Food-Security-in-the-Piedmont-Triad/.

FRAC (2016). How Hungry Is America? Available at: https://www.tiki-toki.com/ timeline/entry/842615/Food-Security-in-the-Piedmont-Triad/.

FRAC (2018). How Hungry Is America?. Available at: https://frac.org/wp-content/ uploads/food-hardship-july-2018.pdf.

Gordon, C., and Hunt, K. (2019). Reform, justice, and Sovereignty: A Food Systems Agenda for Environmental Communication. Environ. Commun. 13 (1), 9-22. doi:10.1080/17524032.2018.1435559

Harter, L. M., Pangborn, S. M., Ivancic, S., and Quinlan, M. M. (2017). Storytelling and Social Activism in Health Organizing. Manag. Commun. Q. 31, 314-320. doi:10.1177/0893318916688090

Heath, R. L., Bradshaw, J., and Lee, J. (2002). Community Relationship Building: Local Leadership in the Risk Communication Infrastructure. J. Public Relations Res. 14, 317-353. doi:10.1207/s1532754xjprr1404_2

Hodgin, C. (2020). Where Students Can Get Free School Meals. WFMY. Available at: https://www.wfmynews2.com/article/news/health/coronavirus/schoolsserving-free-meals-coronavirus-piedmont-triad-nc/83-7blacb44-802f-4ec08411-37bcb0c1ab4d.

Ivancic, S. R. (2020). "No One's Coming to Save Us": Centering Lived Experiences in Rural Food Insecurity Organizing. Health Commun. 36, 1039-1043. doi:10.1080/10410236.2020.1724644

Jovanovic, S., Congdon, M., Jr, Miller, C., and Richardson, G. (2015). Rooting the Study of Communication Activism in an Attempted Book Ban. Partnerships: A J. Service-Learning Civic Engagement 6, 115-135.

Kim, Y.-C., and Ball-Rokeach, S. J. (2006). Civic Engagement from a Communication Infrastructure Perspective. Commun. Theor. 16, 173-197. doi:10.1111/j.1468-2885.2006.00267.x

Kunkle, F., and Ruane, M. E. (2020). Coronavirus Triggers Run on Grocery Stores, with Panic-Buying, Hoarding and Some Fighting, Too. Washington Post. Available at: https://www.washingtonpost.com/dc-md-va/2020/03/13/ coronavirus-triggers-run-grocery-stores-with-panic-buying-hoarding-somefighting-too/.

LeGreco, M., and Douglas, N. (2017). Everybody Eats. Manag. Commun. Q. 31, 307-313. doi:10.1177/0893318916688092

LeGreco, M., and Douglas, N. (2021). Everybody Eats: Communication and the Path the Food Justice. Berkeley: University of California Press.

LeGreco, M., Ferrier, M., and Leonard, D. (2015). "Further Down the Virtual Vines: Managing Community-Based Work in Virtual Public Spaces," in Management and Participation in the Public Sphere (Hershey, PA: IGI Global), 147-169.

LeGreco, M., and Tracy, S. J. (2009). Discourse Tracing as Qualitative Practice. Qual. Inq. 15, 1516-1543. doi:10.1177/1077800409343064

LeGreco, M. (2012). Working with Policy: Restructuring Healthy Eating Practices and the Circuit of Policy Communication. J. Appl. Commun. Res. 40, 44-64. doi:10.1080/00909882.2011.636372

McCullum, C., Desjardins, E., Kraak, V. I., Ladipo, P., and Costello, H. (2005). Evidence-based Strategies to Build Community Food Security. J. Am. Diet. Assoc. 105 (2), 278-283. doi:10.1016/j.jada.2004.12.015

Okamoto, K. E. (2016). "It's like Moving the Titanic:" Community Organizing to Address Food (In)Security. Health Commun. 32, 1047-1050. doi:10.1080/ 10410236.2016.1196517

Pine, A. M., and de Souza, R. (2013). Including the Voices of Communities in Food Insecurity Research: An Empowerment-Based Agenda for Food Scholarship. J. Agric. Food Syst. Community Dev. 3, 71-79. doi:10.5304/ jafscd.2013.034.007

Sampson, R. J., Morenoff, J. D., and Earls, F. (1999). Beyond Social Capital: Spatial Dynamics of Collective Efficacy for Children. Am. Sociological Rev. 64, 633-660. doi: $10.2307 / 2657367$ 
Sandy, M., and Holland, B. A. (2006). Different Worlds and Common Ground: Community Partner Perspectives on Campus-Community Partnerships. Mich. J. Community Serv. Learn. 13, 30-43.

Schraedley, M. K., Bean, H., Dempsey, S. E., Dutta, M. J., Hunt, K. P., Ivancic, S. R., et al. (2020). Food (In)security Communication: A Journal of Applied Communication Research Forum Addressing Current Challenges and Future Possibilities. J. Appl. Commun. Res. 48, 166-185. doi:10.1080/00909882.2020.1735648

Schraedley, M. K., LeGreco, M., and Parry-Giles, T. (2020). Food Insecurity Communication. Communication Matters. The NCA Podcast. Available at: https://www.natcom.org/ncapodcast.

Smith, M., and Mrosla, L. (2010). Guilford County Community Health Assessment 2009-2010. Available at: https://www.guilfordcountync.gov/our-county/humanservices/health-department/health-statistics/2016-community-health-assessment.

Smith, M., and Mrosla, L. (2013). Guilford County Community Health Assessment 2012-2013. Available at: https://www.guilfordcountync.gov/our-county/ human-services/health-department/health-statistics/2016-community-healthassessment.

Smith, M., and Mrosla, L. (2016). Guilford County Community Health Assessment 2015-2016. Available at: https://www.guilfordcountync.gov/our-county/ human-services/health-department/health-statistics/2016-community-healthassessment.

Strauss, A., and Corbin, J. M. (1997). Grounded Theory in Practice. Thousand Oaks, CA: SAGE.

Sy, S. (2020). America's Hunger Crisis Grows More Severe amid Pandemic Recession. PBS. Available at: https://www.pbs.org/newshour/show/americashunger-crisis-grows-more-severe-amid-pandemic-recession.
Tracy, S. J. (2019). Qualitative Research Methods: Collecting Evidence, Crafting Analysis, Communicating Impact. Hoboken, NJ: John Wiley \& Sons.

Wilkin, H. A. (2013). Exploring the Potential of Communication Infrastructure Theory for Informing Efforts to Reduce Health Disparities. J. Commun. 63, 181-200. doi:10.1111/jcom.12006

Wilkin, H. A., Moran, M. B., Ball-Rokeach, S. J., Gonzalez, C., and Kim, Y.-C. (2010). Applications of Communication Infrastructure Theory. Health Commun. 25, 611-612. doi:10.1080/10410236.2010.496839

Conflict of Interest: The authors declare that the research was conducted in the absence of any commercial or financial relationships that could be construed as a potential conflict of interest.

Publisher's Note: All claims expressed in this article are solely those of the authors and do not necessarily represent those of their affiliated organizations, or those of the publisher, the editors and the reviewers. Any product that may be evaluated in this article, or claim that may be made by its manufacturer, is not guaranteed or endorsed by the publisher.

Copyright (c) 2021 LeGreco, Palmer and Levithan. This is an open-access article distributed under the terms of the Creative Commons Attribution License (CC BY). The use, distribution or reproduction in other forums is permitted, provided the original author(s) and the copyright owner(s) are credited and that the original publication in this journal is cited, in accordance with accepted academic practice. No use, distribution or reproduction is permitted which does not comply with these terms. 